\title{
Erratum to: Gemcitabine plus capecitabine (Gem-Cape) biweekly in chemorefractory metastatic colorectal cancer
}

\author{
P. Jiménez-Fonseca $\cdot$ M. P. Solis $\cdot$ M. Garrido $\cdot$ L. Faez $\cdot$ \\ D. Rodriguez $\cdot$ A. L. Ruiz $\cdot$ M. L. Sanchez Lorenzo • \\ E. Uriol $\cdot$ M. D. Menendez $\cdot$ J. M. Viéitez
}

Published online: 6 December 2014

(c) Federación de Sociedades Españolas de Oncología (FESEO) 2014

\section{Erratum to: Clin Transl Oncol}

\section{DOI 10.1007/s12094-014-1243-1}

In the published original article, Table 4, column "Frequency" contains some wrong terms.

The correct table is given here.

The online version of the original article can be found under doi:10.1007/s12094-014-1243-1.

P. Jiménez-Fonseca ( $\square)$ - M. P. Solis · L. Faez · D. Rodriguez ·

A. L. Ruiz · M. L. Sanchez Lorenzo · E. Uriol ·

M. D. Menendez · J. M. Viéitez

Medical Oncology Department, Asturias Central University

Hospital, Carretera de Rubín s/n Finca "La Cadellada",

33011 Oviedo, Asturias, Spain

e-mail: palucaji@hotmail.com

M. Garrido

Hemato-Oncology Department, Pontifical Catholic University,

Santiago, Chile 
Table 4 Studies with gemcitabine and fluoropyrimidines in refractory mCRC

\begin{tabular}{|c|c|c|c|c|c|c|c|c|c|}
\hline Study & Chemotherapy & Dose $\mathrm{mg} / \mathrm{m}^{2} / \mathrm{d}$ & Frequency & $N$ & $\mathrm{RR}(\%)$ & $\begin{array}{l}\text { Disease } \\
\text { control }(\%)\end{array}$ & mPFS & mOS & $\begin{array}{l}\text { Neutrop. } \\
\text { G3/4 (\%) }\end{array}$ \\
\hline \multirow[t]{2}{*}{ Fillos [17] } & Gemcitabine & 750 & Day 1 every 7 days $\times 6$ & 26 & 3.8 & 34.8 & 2.7 & 11.3 & 45 \\
\hline & $5-\mathrm{FU}$ & 450 & Day 1 every 7 days $\times 6$ & & & & & & \\
\hline \multirow[t]{2}{*}{ Pachon [19] } & Gemcitabine & $800-1,250$ & Day 1,15 every 21 & 18 & 0 & 70 & 3.7 & 9.9 & 0 \\
\hline & 5-FU (IC) & 200 & Every 21 days & & & & & & \\
\hline \multirow[t]{2}{*}{ Bitossi [13] } & Gemcitabine & 1,000 & Day $1,8,15$ every 28 & 37 & 10.8 & 61.8 & 4.2 & 8.9 & 8 \\
\hline & 5-FU (IC) & 200 & Every 28 days & & & & & & \\
\hline \multirow[t]{2}{*}{ Madaje Wicz [20] } & Gemcitabine & 900 & Day 1 every 7 days $\times 6$ & 21 & 38 & NR & NR & NR & 11 \\
\hline & $5-\mathrm{FU}$ & 450 & Day 1 every 7 days $\times 6$ & & & & & & \\
\hline \multirow[t]{2}{*}{ Schilsky [15] } & Gemcitabine & 1,000 & Day $1,8,15$ every 28 & 21 & 9.6 & 14.4 & NR & NR & 7.5 \\
\hline & Capecitabine & 1,660 & 21 days every 28 & & & & & & \\
\hline \multirow[t]{2}{*}{ Fernandez [21] } & Gemcitabine & 900 & Day 1 every 14 & 21 & NR & NR & 4 & 9 & NR \\
\hline & Capecitabine & 2,500 & 7 days every 14 & & & & & & \\
\hline \multirow[t]{2}{*}{ Qiu [18] } & Gemcitabine & 1,000 & Day 1,8 every 21 & 12 & 0 & 36.4 & 2.27 & 5.57 & 17 \\
\hline & Capecitabine & 2,000 & 14 days every 21 & & & & & & \\
\hline \multirow[t]{2}{*}{ Current study } & Gemcitabine & 1,000 & Day 1 every 14 & 119 & 7 & 45 & 2.87 & 6.53 & 0 \\
\hline & Capecitabine & 2,000 & 7 days every 14 & & & & & & \\
\hline
\end{tabular}

$d$ day, $R R$ response rate, $m P F S$ median progression-free survival, $m O S$ median overall survival, Neutrop neutropenia 